\title{
The vicinal difluoro motif: The synthesis and conformation of erythro- and threo- diastereoisomers of 1,2-difluorodiphenylethanes, 2,3-difluorosuccinic acids and their derivatives
}

\author{
David O'Hagan ${ }^{* 1}$, Henry S. Rzepa ${ }^{2}$, Martin Schüler ${ }^{1}$
}

and Alexandra M. Z. Slawin ${ }^{1}$

\section{Full Research Paper}

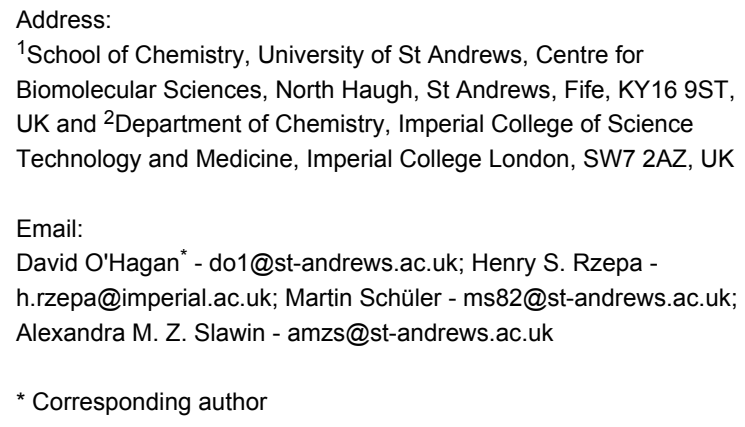

${ }^{1}$ School of Chemistry, University of St Andrews, Centre for Biomolecular Sciences, North Haugh, St Andrews, Fife, KY16 9ST, UK and ${ }^{2}$ Department of Chemistry, Imperial College of Science Technology and Medicine, Imperial College London, SW7 2AZ, UK

Email:

David O'Hagan* - do1@st-andrews.ac.uk; Henry S. Rzepa h.rzepa@imperial.ac.uk; Martin Schüler - ms82@st-andrews.ac.uk; Alexandra M. Z. Slawin - amzs@st-andrews.ac.uk

* Corresponding author

\begin{abstract}
Beilstein Journal of Organic Chemistry 2006, 2, No. 19 doi:10.1186/1860-5397-2-19

Received: 10 August 2006

Accepted: 02 October 2006

Published: 02 October 2006

(c) 2006 O'Hagan et al; licensee Beilstein-Institut.

License and terms: see end of document.
\end{abstract}

\begin{abstract}
Background

It is well established that vicinal fluorines (RCHF-CHFR) prefer to adopt a gauche rather than an anti conformation when placed along aliphatic chains. This has been particularly recognised for 1,2-difluoroethane and extends to 2,3-difluorobutane and longer alkyl chains. It follows in these latter cases that if erythro and threo vicinal difluorinated stereoisomers are compared, they will adopt different overall conformations if the fluorines prefer to be gauche in each case. This concept is explored in this paper with erythro- and threo- diastereoisomers of 2,3-difluorosuccinates.
\end{abstract}

\section{Results}

A synthetic route to 2,3-difluorosuccinates has been developed through erythro- and threo- 1,2-difluoro-1,2-diphenylethane which involved the oxidation of the aryl rings to generate the corresponding 2,3-difluorosuccinic acids. Ester and amide derivatives of the erythro- and threo-2,3-difluorosuccinic acids were then prepared. The solid and solution state conformation of these compounds was assessed by X-ray crystallography and NMR. Ab initio calculations were also carried out to model the conformation of erythroand threo-1,2-difluoro-1,2-diphenylethane as these differed from the 2,3-difluorosuccinates.

\section{Conclusion}

In general the overall chain conformations of the 2,3-difluorosuccinates diastereoisomers were found to be influenced by the fluorine gauche effect. The study highlights the prospects of utilising the vicinal difluorine motif (RCHF-CHFR) as a tool for influencing the conformation of performance organic molecules and particularly tuning conformation by selecting specific diastereoisomers (erythro or threo). 


\section{Background}

Of the 298,876 registered fluorinated structures in the Beilstein Chemical Database (for 2005) only 279 compounds contain a genuine vicinal difluoro motif-CHF-CHF- and only 12 crystal structures of this motif are deposited in the Cambridge Structure Data Base. The relatively rare presence of this motif may partly be attributed to the difficulty of their selective synthesis. It remains a synthetic challenge to prepare vicinal difluorocompounds efficiently and particularly in a stereoselective manner. There are attractive reasons to explore this motif. It is well known that the conformation of 1,2-difluoroethane is influenced by the fluorine gauche effect, where the fluorines prefer to be gauche rather than anti to each other [1]. This preference extends to 2,3-difluorobutane [2], and we have shown that erythro- and threo-9,10 difluorostearic acids have very different physical properties [3], the origin of which appears to lie in the different conformational preferences associated with the vicinal difluoro- motif for each diastereoisomer. Early synthetic methods to vicinal difluoro compounds have involved direct fluorination of alkenes with for eg. elemental fluorine $\left(\mathrm{F}_{2}\right)$ [4] or $\mathrm{XeF}_{2}$ [5]. Such methods however are either difficult to carry out in a standard laboratory environment or they suffer from very poor stereoselectivity. The direct conversion of vicinal diols to vicinal difluorides has been explored with some success. For example both erythro and threo stereoisomers of dimethyl 2,3-difluorosuccinic acid were obtained either from methyl esters of the L-tartrate 1 or the meso-tartrate 1 by treatment with $\mathrm{SF}_{4} / \mathrm{HF}$ (Scheme 1) [6,7]. Conversion to the product erythro-2 proved efficient (97\%) but that to threo-2 was poor $(23 \%)$ due to competing elimination. The preparation of the erythro isomer of $\mathbf{2}$ is attractive on a large scale although $\mathrm{SF}_{4}$ has to be used with care and it is not amenable to reactions on a small scale. Our attempts to replace $\mathrm{SF}_{4}$ with DAST failed in trying to develop an analogous small scale laboratory process. Deoxofluor is finding use in the stereoselective conversion of<smiles>CC(=O)[C@H](O)[C@@H](O)C(C)=O</smiles><smiles>COC(=O)C(F)C(F)C(C)=O</smiles><smiles>CC(=O)[C@H](O)[C@@H](O)C(C)=O</smiles><smiles>COC(C)C(F)C(F)C(C)=O</smiles>

Scheme 1: Synthesis of vicinal dimethyl difluorosuccinates. The conversion of the tartrates 1 with $\mathrm{SF}_{4}$ and $\mathrm{HF}[6,7]$.

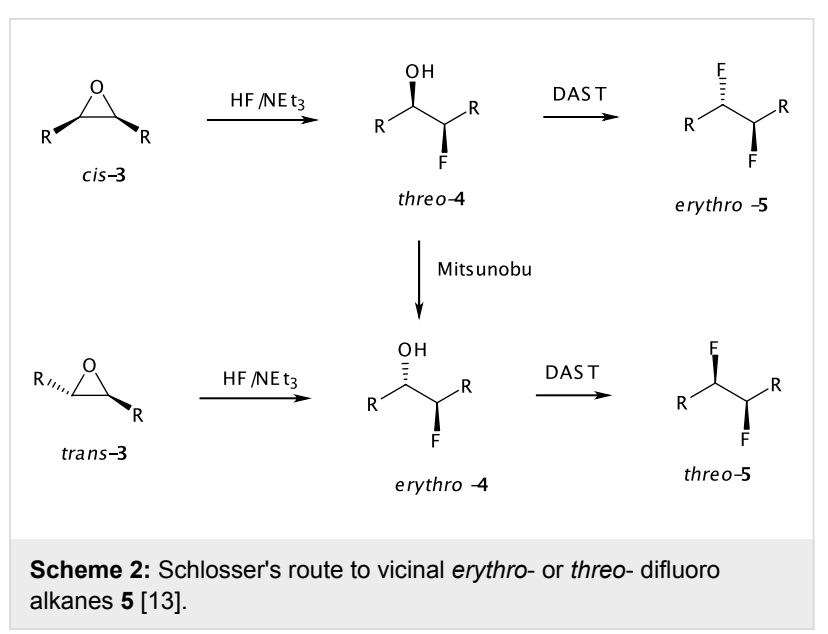

vicinal diols to vicinal difluorocompounds and seems less prone to elimination than DAST [8]. In addition, Deoxofluor has been reported to be thermally more stable than related aminosulfur trifluoride reagents which allows the conversions to be carried out safely at elevated temperatures $[9,10]$. The stereoselective conversion of vicinal ditriflates to vicinal difluorides by treatment with TBAF has also been reported, particularly for the synthesis of 3,4-difluoropyrrolidine ring systems, and these reactions are finding currency in pharmaceutical products [11, 12]. Schlosser et al. [13] have developed the most practical and straightforward method to access a variety of erythro- or threovicinal difluoro compounds in a diastereoselective manner, using either cis- or trans- epoxides 3 obtained directly from either the $Z$ - or the $E$ - alkenes. (Scheme 2). Ring opening of the epoxides 3 with HF-amine reagents generate the corresponding threo- and erythro- fluorohydrins $\mathbf{4}$ in largely a stereoselective manner. The resulting fluorohydrins $\mathbf{4}$ can then be converted to the erythro- or the threo- vicinal difluoro compounds 5 with reagents such as DAST $[9,10]$ or Deoxofluor $[8,14]$, although elimination products often compete with fluoride substitution depending on the nature of the substrate.

Vicinal difluoro compounds have been prepared by halo(bromo/iodo)fluorination of alkenes followed by halide substitution with silver fluoride [15]. The reaction has been applied to a variety of alkenes some of which (eg 6-9) are illustrated in Scheme 3.

We were interested in accessing diastereomerically pure samples of erythro- and threo- 2,3-difluorosuccinic acids 19. The preparation of stereoisomers of 2,3-difluorosuccinic acids, has involved conversions of tartaric acids (esters) [6,7], as described above in Scheme 1. Other approaches have involved the direct fluorination of fumaric acid [16] and the catalytic hydrogenation of 2,3-difluoromaleic acid [7], but these 


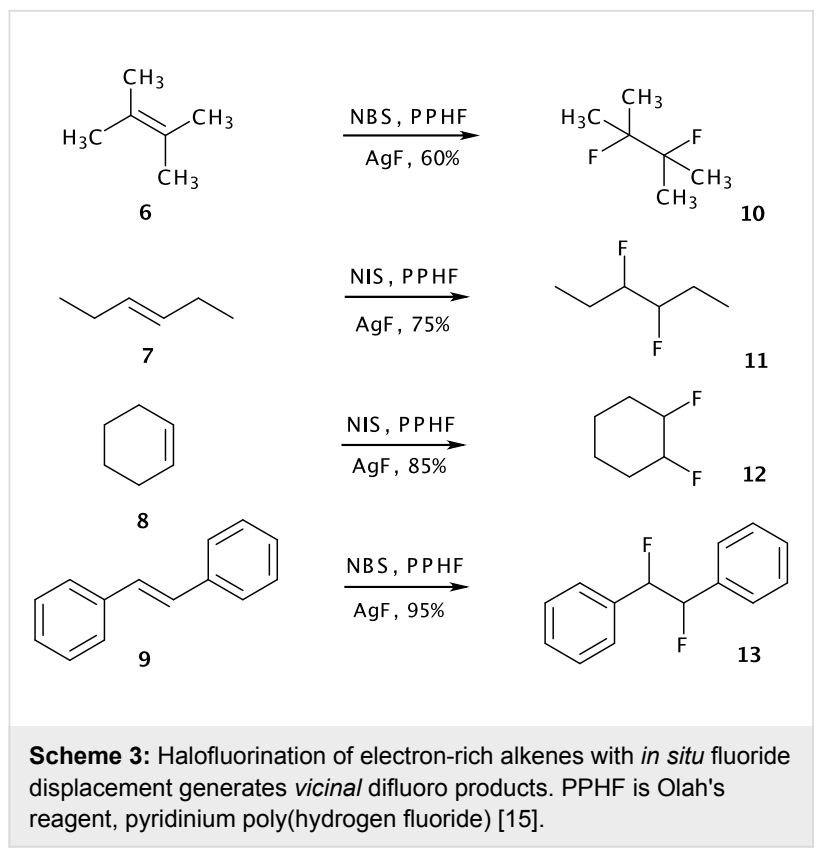

processes result in significant by-product formation and gave only poor yields of the desired products. Our alternative approach chose to explore the oxidation of the aromatic rings of erythro- and threo- diastereoisomers of 1,2-diphenyl-1,2difluoroethane $\mathbf{1 3}$, exploiting the ability of the phenyl group to act as a latent carboxylic acid [17]. This paper describes these studies and we report the solid and solution state conformation of the erythro- and threo-diastereoisomers of $\mathbf{1 3}$ and the resultant 2,3-difluorosuccinic acid stereoisomers and some of their derivatives. Some of these results have recently been communicated [18]. The study suggests that the vicinal fluorine gauche effect can have a significant influence on the conformation of the 1,2-difluorosuccinates.

\section{Results and Discussion Synthesis of erythro- and threo- 1,2-diphenyl- 1,2-difluoroethanes 13}

Stilbene 9 is readily converted to its bromofluoro adduct by treatment with NBS and pyridine:HF following Olah's method [19] (Scheme 4).

In our hands product $\mathbf{1 4}$ was generated with a diastereoselectivity of $94 \%$. The predominant anti stereochemistry of $\mathbf{1 4}$ was established from the coupling constants of the olefin products obtained after a dehydrobromination reaction. The elimination of hydrogen bromide from such $\beta$-fluorobromides had been explored previously, and the reaction proceeds in a stereospecific manner to generate either $E$ or $Z$ fluoroalkene products [20]. Accordingly treatment of $\mathbf{1 4}$ with potassium tertbutoxide in a refluxing solution of hexane or pentane lead to the exclusive formation of the $E$-alkene $\mathbf{1 5}$ as judged by the ${ }^{3} J_{\mathrm{HF}}$

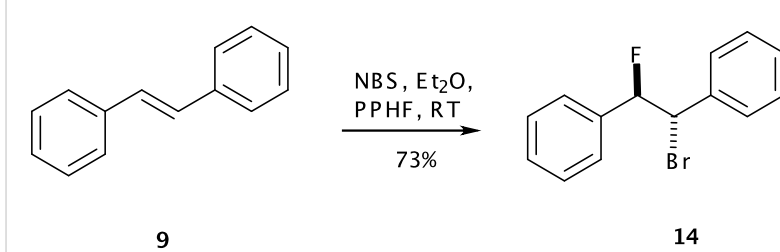

Scheme 4: Bromofluorination of stilbene [19].

coupling constant of $21.1 \mathrm{~Hz}$ obtained from ${ }^{19} \mathrm{~F}-\mathrm{NMR}$. This is indicative of a stereospecific anti-elimination of hydrogen bromide from 14 to generate 15 with a cisoid relationship between $\mathrm{H}$ and $\mathrm{F}$, rather than compound $\mathbf{1 6}$ which would have a trans relationship and a much larger ${ }^{3} J_{\mathrm{HF}}$ coupling constant $(\sim 30 \mathrm{~Hz})$, and reinforces the stereochemical assignment made to $\mathbf{1 4}$ as illustrated in Scheme 5 [21].

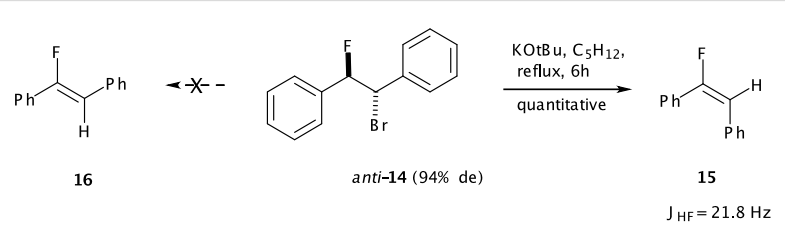

Scheme 5: Treatment of anti-14 with base generated the E-fluorostilbene 15 by an anti elimination mechanism.

Substitution of the bromine in anti-14 with fluorine was accomplished by treatment with $\mathrm{Ag}(\mathrm{I}) \mathrm{F}$ in acetonitrile in the dark. Under these conditions, the substitution proceeds smoothly to erythro-13 but only in $56 \%$ de indicating a significant loss of stereochemical control during the reaction. The predominant stereochemical outcome of the fluorine substitution reaction suggests a double inversion mechanism as the major erythro-13 isomer must arise by replacement of the bromine of anti-14 by fluorine with an overall retention of configuration. Various examples of anchimeric assistance by phenyl groups have been reported [22] and in this case a carbocation is most reasonably generated which finds benzylic as well as anchimeric stabilisation via phenonium ring formation 18 with the $\beta$-phenyl group as illustrated in Scheme 6.

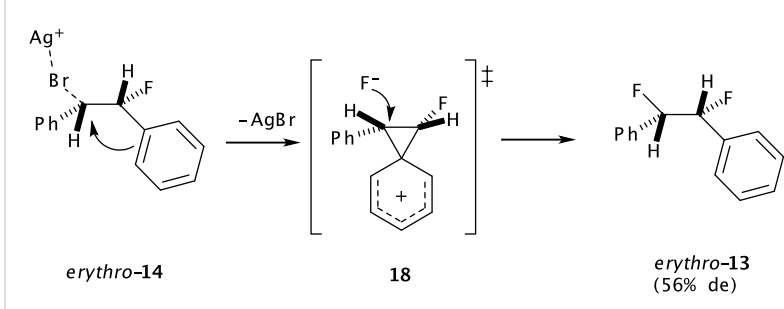

Scheme 6: Hypothesis for the predominent retention of configuration during fluoride substitution via phenonium intermediate 18. 


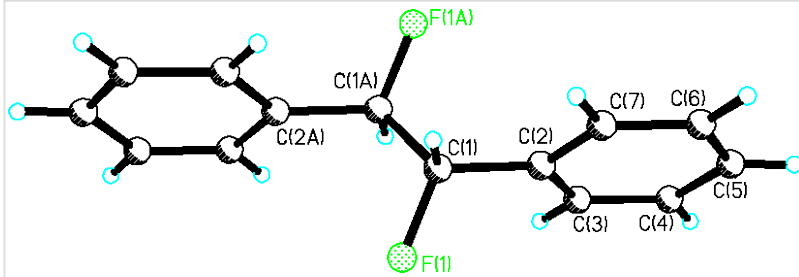

Figure 1: Crystal structure of erythro-13.

Isolation of the minor threo-13 isomer required careful chromatography. In order to improve the synthesis of threo-13 a reaction with cis-stilbene 17 was investigated. The one pot process with NBS, PPHF and Ag(I)F again proceeded smoothly however it also gave erythro-13 as the major product of the reaction, although with a reduced diastereoisomeric ratio $(47 \%$ de) more suitable for threo- $\mathbf{1 3}$ isolation. The bias towards erythro- $\mathbf{1 3}$ in this case is clearly a result of internal rotation about the central carbon-carbon bond, to relieve a steric clash between the vicinal phenyl groups, after initial formation of an intermediate bromonium ion $\mathbf{1 8}$ as illustrated in Scheme 7.

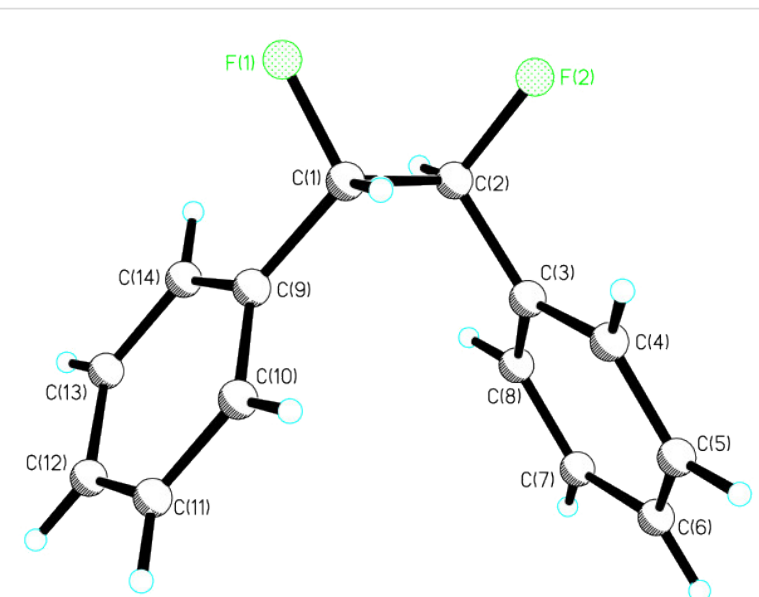

Figure 2: X-ray structure of threo-13
Erythro 13 was readily purified after several crystallisations whereas isolation of the threo isomer of $\mathbf{1 3}$ was more challenging. Partial separation of threo-13 was achieved by means of preparative thin layer chromatography. The enriched diasteroisomeric mixture could be crystallised to purity and crystals suitable for X-ray structure analysis were obtained (Figure 1). In the solid state erythro-13 adopts a conformation in which the phenyl substituents are anti to each other, with a Ph-C-C-Ph torsion angle of $180^{\circ}$. As a result the $\mathrm{C}-\mathrm{F}$ bonds also align anti with respect to each other with a F-C-C-F torsion angle also close to $180^{\circ}$.

A stereochemical mixture enriched in favour of threo-13 was crystallised to purity and a suitable crystal was used for X-ray structure analysis. The resultant structure is shown in Figure 2.

The most obvious feature of this structure is the perhaps unexpected gauche relationship between the phenyl rings which places the fluorine atoms also gauche to each other. This superficially suggests that the fluorine gauche effect is over-riding any steric repulsion between the phenyl rings. To explore the significance of these solid state conformations further, NMR solution studies and ab initio analysis, exploring the preferred conformations for each of the diastereoisomers was carried out.

\section{NMR studies on erythro- and threo-13}

The most obvious feature in the ${ }^{1} \mathrm{H}$ - and ${ }^{19} \mathrm{~F}$ - NMR spectra of the diastereoisomers of $\mathbf{1 3}$ is the coupling pattern from the AA'XX' spin system (Figure 3). Due to the chemical equivalence but magnetic non-equivalence of the $\mathrm{F}$ and $\mathrm{H}$ atoms a second-order spectrum is generated in each case.

Measuring of coupling constants from such second-order spectra has been described by Abrahams et. al. [23] although the analysis requires an intuitive fitting of values to specific coupling relationships. These deduced values are tabulated in Figure 3c. The large values of $45.2 \& 47.2 \mathrm{~Hz}$ clearly correlate to the geminal ${ }^{2} J_{\mathrm{HF}}$ coupling, and the values of $15.2 \& 14.1 \mathrm{~Hz}$ to the vicinal ${ }^{3} J_{\mathrm{HF}}$ coupling. The smaller coupling constant of

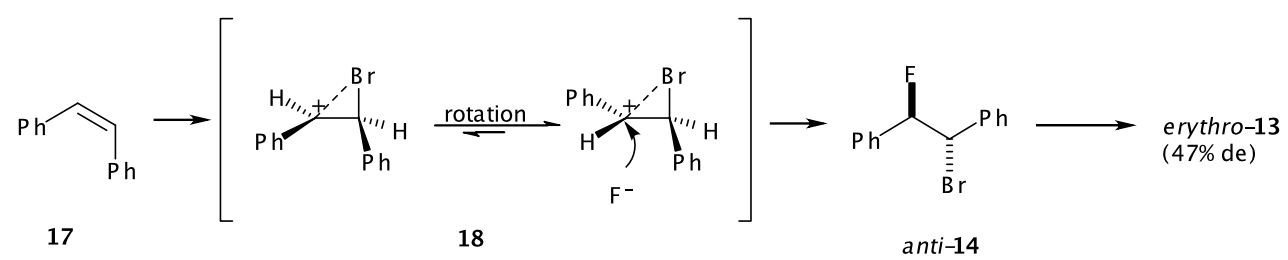




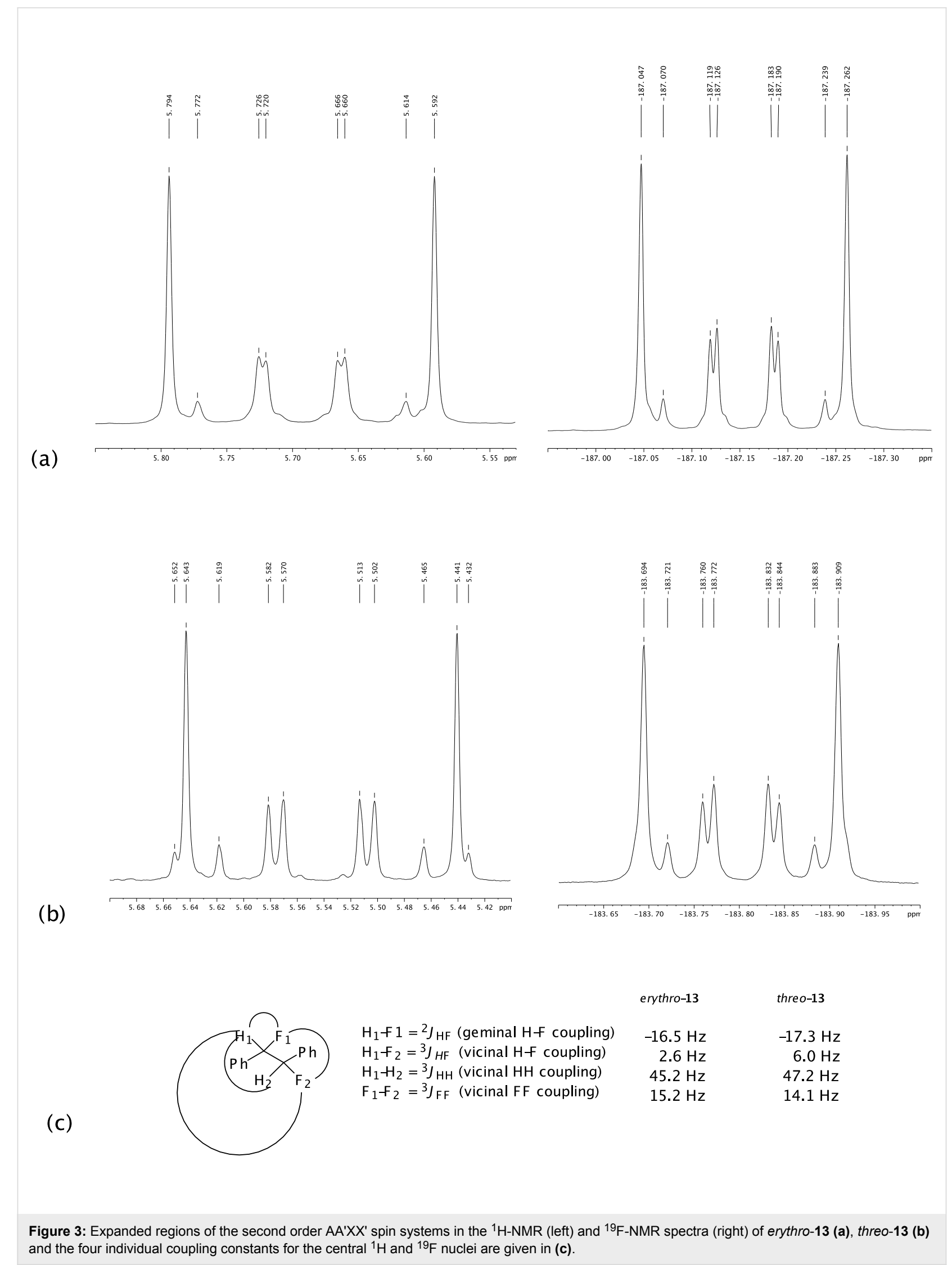




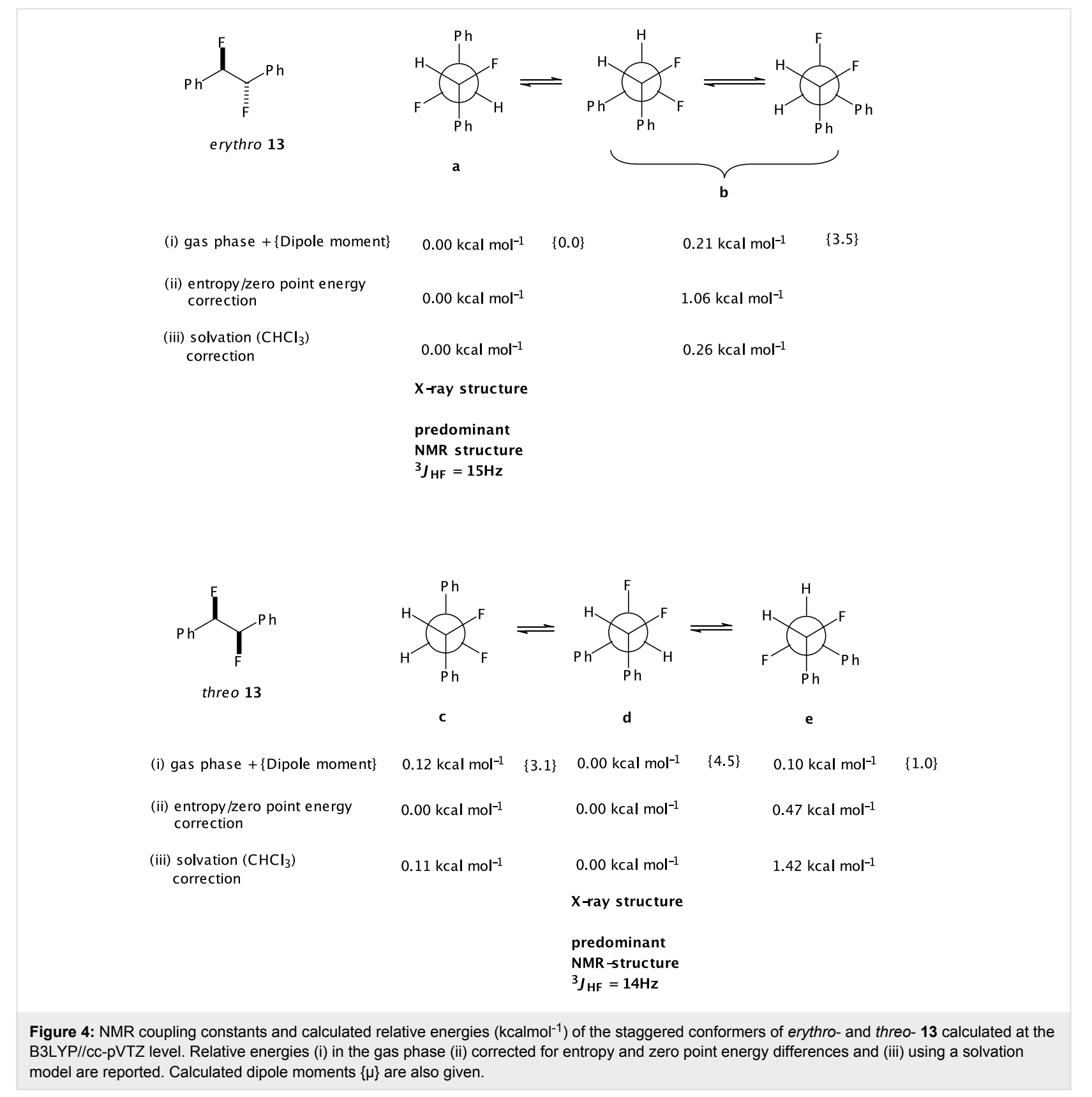

$2.6 \& 6.0 \mathrm{~Hz}$ most appropriately correlate to the ${ }^{3} J_{\mathrm{HH}}$ couplings, and thus, the value of $-16.5 \&-17.3 \mathrm{~Hz}$ is assigned to the vicinal ${ }^{3} J_{\mathrm{FF}}$ coupling. The ${ }^{19} \mathrm{~F}$ NMR spectrum can similarly be assigned in each case and reinforced these values. The magnitude of the different vicinal NMR coupling constants can be rationalised in terms of rotational isomerism of the individual diastereoisomers. Only the three staggered conformations for erythro- and threo- $\mathbf{1 3}$ are considered (Figure 4).

It is not obvious from the NMR data which of $\mathbf{a}$ or $\mathbf{b}$ is the favoured solution conformation for the erythro isomer. We infer a significant contribution from rotamer $\mathbf{b}$ where the $\mathrm{C}-\mathrm{H}$ bonds are gauche on the basis of the small ${ }^{3} J_{\mathrm{HH}}$ value $(2.6 \mathrm{~Hz})$, however the relatively small ${ }^{3} J_{\mathrm{HF}}$ value $(15 \mathrm{~Hz})$ suggests two $\mathrm{C}-\mathrm{H}$ and $\mathrm{C}-\mathrm{F}$ gauche relationships implying a contribution from rotamer a. Rotamer a most closely resembles the X-ray structure for erythro-13 shown in Figure 1. The situation is much clearer for threo-13. The relatively large ${ }^{3} J_{\mathrm{HH}}$ coupling constant $(6.0 \mathrm{~Hz})$ and the small ${ }^{3} J_{\mathrm{HF}}$ coupling constant $(14 \mathrm{~Hz})$ suggests a significant population of rotatmer $\mathbf{d}$. This isomer has the vicinal C-H bonds anti to each other and both of the $\mathrm{C}-\mathrm{F} / \mathrm{C}-\mathrm{H}$ and C-F/C-F bonds gauche. This is also the preferred conformation for this compound in the solid state (X-ray structure in Figure 2). 


\section{Conformational energy calculations on erythro and threo-13}

Due to the ambiguous solution state study particularly for erythro-13, ab initio calculations were carried out at the B3LYP//cc-pVTZ level exploring absolute energies of the three staggered conformers of both erythro- and threo- 13 [24,25]. The geometries were optimized at this level for a gas phase model, and corrected for entropy and zero-point energy differences at this level. A separate solvation correction (chloroform) was applied using a continuum model (PCPM) and the larger cc-pV5Z basis set (using pVTZ geometries). Chloroform was studied in an attempt to relate the calculated values to the NMR solution conformations (vide infra). The relative energy data and dipole moments for each diastereoisomer are presented in Figure 4. The calculated conformations and energies can be viewed at http://www.ch.ic.ac.uk/rzepa/ohagan/ (see Supporting Information File 2).

Of the three staggered conformers of the erythro-13 isomer two are enantiomeric and have identical energies thus analysis of erythro-13 is reduced to a comparison of the energies of conformers $\mathbf{a}$ and $\mathbf{b}$. Conformer a emerges as the more stable in the gas phase, with this stability originating predominantly from entropy and zero-point energy corrections $(1.06 \mathrm{kcal} / \mathrm{mol})$. This is also the conformer that most closely represents the X-ray structure (Figure 1). The solvent correction (which takes into account free energy differences associated with the solvent cavity, but does not allow for free energy differences arising from vibrational terms) does not alter the relative energies of a and $\mathbf{b}$, despite a having a zero dipole moment and $\mathbf{b}$ having a relatively large value (3.5D) [26]. Although the more polar b should perhaps gain more from electrostatic solvation, it has a smaller solvent accessible surface area $\left(239 \mathrm{~A}^{2}\right.$ vs $246 \mathrm{~A}^{2}$ for a) and these two appear to cancel in their overall effect on the relative energies. Our best estimate of the relative stability of a and $\mathbf{b}$ is about $1.0 \mathrm{kcal} / \mathrm{mol}$ in favour of the former as noted above. Thus structure a does not conform to a fluorine gauche effect and appears to be dominated by solvation of the trans relationship of the aryl rings and the zero dipole moment, although the smaller ${ }^{3} J_{\mathrm{HH}}$ coupling of $2.6 \mathrm{~Hz}$ and the slightly larger ${ }^{3} J_{\mathrm{HF}}$ coupling of $15 \mathrm{~Hz}$ in the NMR, measurement does suggest some contribution of conformer $\mathbf{b}$ in solution.

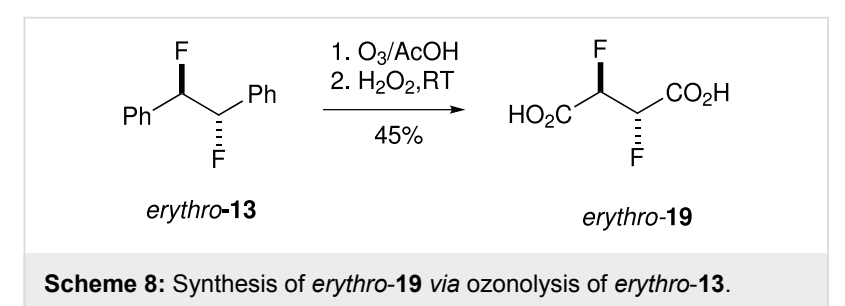

Scheme 8: Synthesis of erythro-19 via ozonolysis of erythro-13.
The threo-13 isomer has three distinct staggered conformations; c, $\mathbf{d}$ and e. Computationally, this requires modelling the subtle balance between the correlation effects due to gauche fluorine atoms and those due to gauche phenyl rings. In the gas phase (entropy and zero energy corrected) conformers $\mathbf{c}$ and $\mathbf{d}$ are isoenergetic. The dipole moments for these conformers vary significantly, with $\mathbf{d}>\mathbf{c}>\mathbf{e}$. As with the erythro isomer, the greater solvation for $\mathbf{d}$ is partially offset by a smaller solvent-accessible surface ( 238 vs $247 \AA^{2}$ for c). Although d is slightly favoured in this model (by $0.11 \mathrm{kcal} / \mathrm{mol}$ ), this is significantly smaller than the NMR estimate and may reflect a limitation of the solvation model. Taking all of the data together (theory, X-ray and NMR) conformer $\mathbf{d}$ appears to be the most favoured conformer for threo-13 with both the fluorine and the phenyl rings gauche, despite its larger dipole moment.

\section{2,3-Difluorosuccinic acids 19}

The synthesis of the 2,3-difluorosuccinic acid diastereoisomers 19 was explored by the oxidation of the aryl rings of $\mathbf{1 3}$ to carboxylic acids. Oxidative degradation of aromatic rings has been achieved by $\mathrm{RuCl}_{3} / \mathrm{NaIO}_{4}$ oxidation [17] however this method proved unsatisfactory in our hands and lead to poor conversions and a complex product mixture. As an alternative strategy ozonolysis in acetic acid, with a hydrogen peroxide work-up was explored [27,28], and this proved successful as illustrated in Scheme 8. For example, reaction of a 4:1 mixture of erythro- and threo- $\mathbf{1 3}$ led to the formation of $\mathbf{1 9}$ also in a 4:1 ratio of diastereoisomers. Erythro 2,3-difluorosuccinic acid 19 was obtained in a modest yield as a single stereoisomer from a stereochemically pure sample of the erythro 13. A crystal of erythro-19 suitable for X-ray analysis was obtained after sublimation, and the resultant structure is shown in Figure 5.

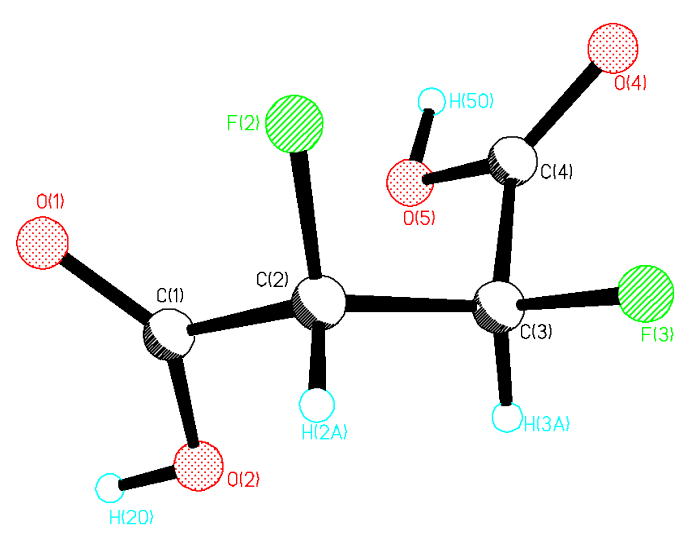

Figure 5: X-ray structure of erythro-19. 
In the X-ray crystal structure of erythro-19 both of the carboxylic acid carbonyl oxygens adopt a syn periplanar conformation with respect to the C-F bonds. In the crystal packing, the carboxylate groups of two neighbouring molecules are hydrogen bonded and this clearly determines the three dimensional structure of the unit cell. The threo-19 diastereoisomer was prepared by a similar aryl oxidation reaction on a diastereomerically pure sample of threo-13 and this allowed crystallisation of a sample of racemic threo-19. The X-ray structure in Figure 6 shows the molecule in an extended chain conformation with both of the C-F bonds gauche to each other. One molecule of water is bound for every succinic acid molecule and this water clearly participates in hydrogen bonding to the carboxylic acid groups.

The major by-product of the ozonolysis reaction of $\mathbf{1 3}$ was the vicinal difluorophenylpropionic acid $\mathbf{2 0}$ as a mixture of stereoisomers. The compound was purified by esterification with methanol to generate esters 21 . These diastereoisomers could be separated by chromatography and then hydrolysis was achieved under acidic conditions, followed by recrystallisation as illustrated in Scheme 9 to generate racemic, but diastereomerically pure samples of erythro- and threo- $\mathbf{2 0 .}$

The ${ }^{3} \mathrm{~J}_{\mathrm{HH}}$ coupling constants of esters 21 remain small (2.8-3.6 $\mathrm{Hz}$ ) and indicate a gauche relationship between these vicinal hydrogens as summarised in Figure 7. It follows that in each case the fluorines will be predominantly gauche to each other.

The observed values for erythro- 21 report a maximal ${ }^{3} J_{\mathrm{HF}}$ coupling constant for the $\beta$-fluorine $(28 \mathrm{~Hz}$ ), but an intermediate one for the $\alpha$-fluorine $(20.8 \mathrm{~Hz})$. This suggests a conform-

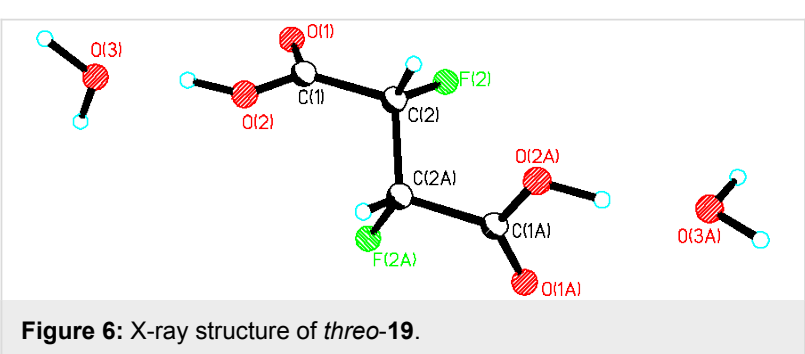

ational preference for rotamer $\mathbf{c}$, which has a gauche vicinal fluorine relationship, over a (Figure 8). For the threo- 21 isomer, there are two vicinal ${ }^{3} J_{\mathrm{HF}}$ couplings of similar and large magnitude (26.3 and $23.6 \mathrm{~Hz}$ ) suggesting that rotamer d, with two trans ${ }^{3} J_{\mathrm{HF}}$ relationships and again with the fluorines gauche, is the most significant contributor to the solution conformation.

In the solid state structure of threo-21 in Figure 9, the C-F bonds adopt a gauche relationship and the phenyl and ester groups are anti to each other. This is consistent with the preference for rotamer $\mathbf{d}$ found in solution. Attempts to crystallise erythro-21 as its free carboxylic acid resulted only in the formation of amorphous material and thus a comparison of solution and solid state structures was not possible for this isomer.

\section{Amides of 2,3-difluorosuccinic acid}

It was an objective of this research to explore the conformational preferences of amides of 2,3-difluorosuccinamides, particularly as we have previously noticed a conformational presence in $\alpha$-fluoroamides [29], where the C-F bond aligns anti and planar to the amide carbonyl as illustrated in Figure 10. This adds an additional conformational constraint to these amides with a barrier to rotation around the $\mathrm{C}(\mathrm{CO})-\mathrm{C}(\mathrm{F})$ bond of around 7-8 kcal mol-1. The preference of the C-F bond in $\alpha$-fluoroamides to align anti periplanar to the carbonyl bond can be rationalized in terms of $\mathrm{C}-\mathrm{F}$ bond and amide dipole relaxation as well as N-H...F hydrogen bonding [30].

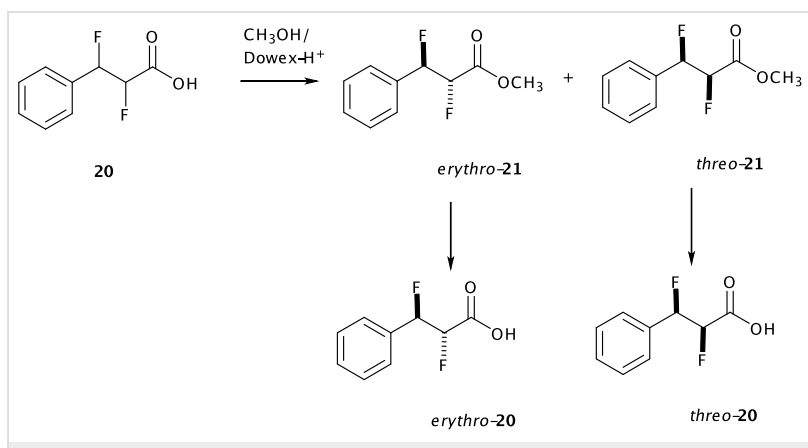

Scheme 9: Strategy for the preparation of diastereoisomers of erythroand threo- 20.
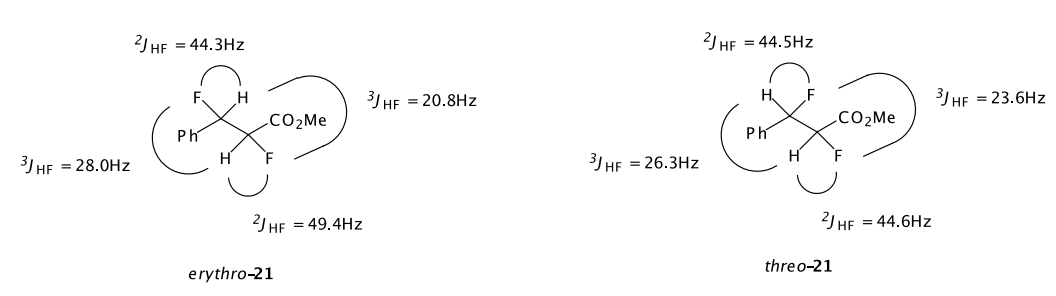

Figure 7: NMR $\left(\mathrm{CDCl}_{3}, \mathrm{RT}\right)$ coupling constants of erythro- and threo- 2,3-difluoro-3-phenylpropionates 21. 
<smiles>CC(=O)[C@H](F)[C@H](F)c1ccccc1</smiles>

erythro-21<smiles>C=CC1c2ccc(c(F)c2)C(=O)C1F</smiles>

a<smiles>CCCCC1CC2C(=O)C1C(c1ccccc1)C(F)C2F</smiles>

b

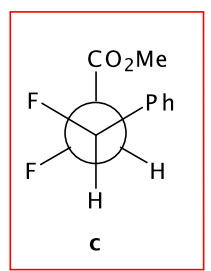<smiles>C=CO[C@H](F)[C@H](F)[C@H](F)c1ccccc1</smiles>

Figure 8: Newman projections showing the staggered conformations of erythro- and threo- 21.
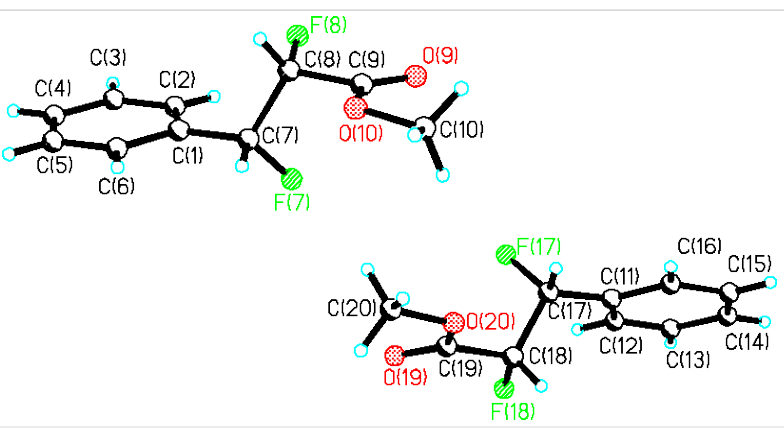

Figure 9: X-ray structure of methyl threo- 21

The solution and solid state structures of 2,3-difluorosuccinate benzylamides 22 have been evaluated. These compounds were prepared by a straightforward EDCI amide coupling between benzylamine and 2,3-difluorosuccinate $\mathbf{1 9}$ as shown in Scheme 10.

The diasteroisomers of $\mathbf{2 2}$ were separated by silica gel chromatography and recrystallisation of each allowed their X-ray structures to be compared. The structure of erythro-22 is illustrated in Figure 11.

Erythro- 22 adopts an extended conformation of the main chain in which the C-F bonds are anti with respect to each other. In that conformation the large benzyl substituents point in opposite

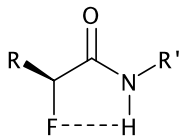

Figure 10: The preferred conformation of $\alpha$-fluoroamides has the C-F and amide carbonyl anti-planar [29,30].

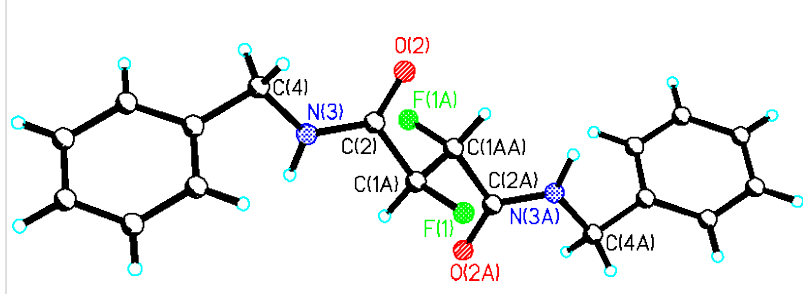

Figure 11: X-ray structure of erythro-22

directions. The $\alpha$-fluoroamide groups tend towards a syn-planar C-F...N-H conformation as it is typical for this functional group (Figure 10) with the $\mathrm{C}-\mathrm{F}$ bonds only $23^{\circ}$ off the plane. The carbonyls point in opposite directions and thus intramolecular hydrogen bonding is not possible. There is however strong intermolecular hydrogen bonding between the amide hydrogen and the carbonyl oxygen of adjacent molecules which is dominating the unit cell structure (Figure 12).<smiles>O=C(O)C(F)C(F)C(=O)O</smiles>

19

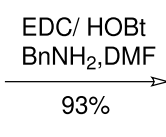

$93 \%$<smiles>O=C(NCc1ccccc1)C(F)C(F)C(=O)NCc1ccccc1</smiles>

22<smiles>O=C(NCc1ccccc1)C(F)C(F)C(=O)NCc1ccccc1</smiles>

22 


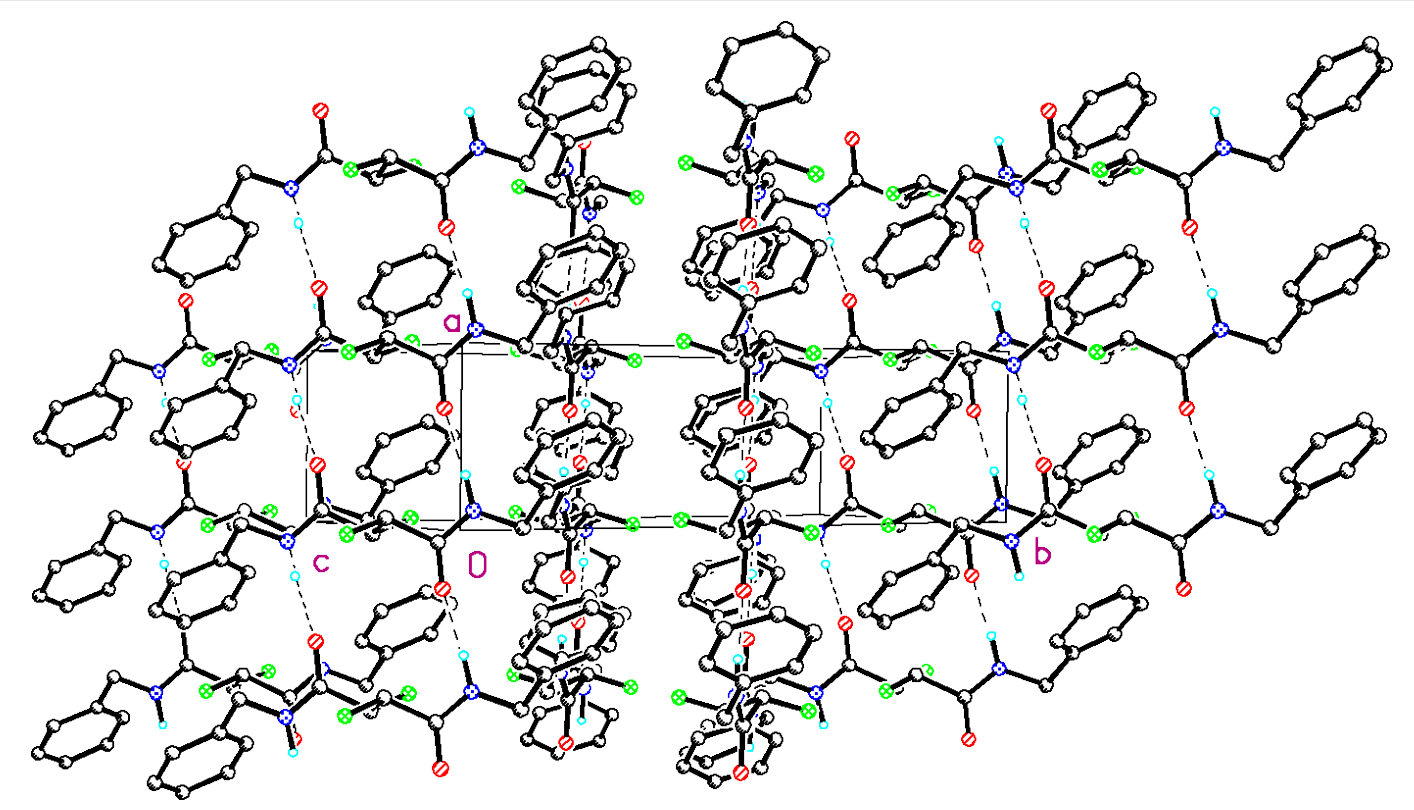

Figure 12: Crystal packing of erythro-22 clearly indicating intermolecular hydrogen bonding.

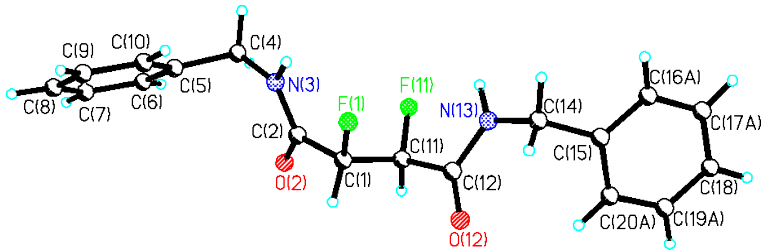

Figure 13: X-ray structure of threo-22.

These intermolecular interactions apparently over-ride the stereoelectronic preference for the gauche arrangement of the $\mathrm{C}-\mathrm{F}$ bonds, which is observed in solution (vide infra). So we conclude that the solid and solution state structures of erythro22 are quite different. By comparison with erythro-22, the crystal structure of threo- 22 in Figure 13 shows both C-F bonds perfectly syn planar with respect to the amide N-H bonds, consistent with the typical planar arrangement of the $\alpha$-fluoroamide group (Figure 10). The vicinal fluorines are gauche to each other. In this case the solution and solid state structures appear to be much more similar.

In a recent Communication [18] we have reported the synthesis of peptides with 2,3-difluorosuccinic acid cores and revealed that such compounds adopt different conformations as a consequence of either the erythro or threo vicinal fluorine stereochemistry. That study highlighted both the solution and solid state conformations of the erythro and threo diastereoisomers of the bis- $(S)$-phenylalanine amides $\mathbf{2 3}$ as shown in Figure 14. The solution and solid state structures reinforced each other and the two diastereoisomers of $\mathbf{2 3}$ had preferred conformations where the fluorine atoms were again gauche to each other. This however gave very different shapes to the backbone connectivity in each diastereoisomer as illustrated in Figure 14.

\section{NMR studies of vicinal difluoro diastereoi- somers}

The vicinal difluorosuccinates again give rise to second order NMR spectra due to the chemical equivalence but magnetic non equivalence of the fluorine and $\mathrm{CHF}$ methine hydrogen atoms similar to Figure 3. A comparison of the ${ }^{3} J_{\mathrm{HH}}$ and ${ }^{3} J_{\mathrm{HF}}$ coupling constants is outlined for the vicinal difluorosuccinate diastereoisomers 19-22, 24 and the 1,2-difluoro-1,2-diphenylethanes $\mathbf{1 3}$ in Figure 15.

Interestingly, the ${ }^{3} J_{\mathrm{HF}}$ coupling constants are very similar to each other within each diastereoisomeric series and are essentially independent of the nature of the substituents attached to the carboxylate group. The only significant exception are the diastereoisomers of 1,2-difluoro-1,2-diphenylethanes 13 which have already been discussed in detail. For the 2,3-difluorosuccinate derivatives 19-22,24 the threo stereoisomers have larger ${ }^{3} J_{\mathrm{HF}}$ coupling constants than the erythro stereoisomers.

In order to interpret the data in Figure 15, it is again useful to consider the staggered conformations of each threo and erythro diastereoisomer as shown in Figure 16. Each rotational isomer has two ${ }^{3} J_{\mathrm{HF}}$ and two ${ }^{3} J_{\mathrm{HH}}$ coupling constants the overall magnitude of each being an average of the two. 
<smiles>CC(=O)C(Cc1ccccc1)NC(=O)C(F)C(F)C(=O)NC(Cc1ccccc1)C(C)=O</smiles>

threo-23
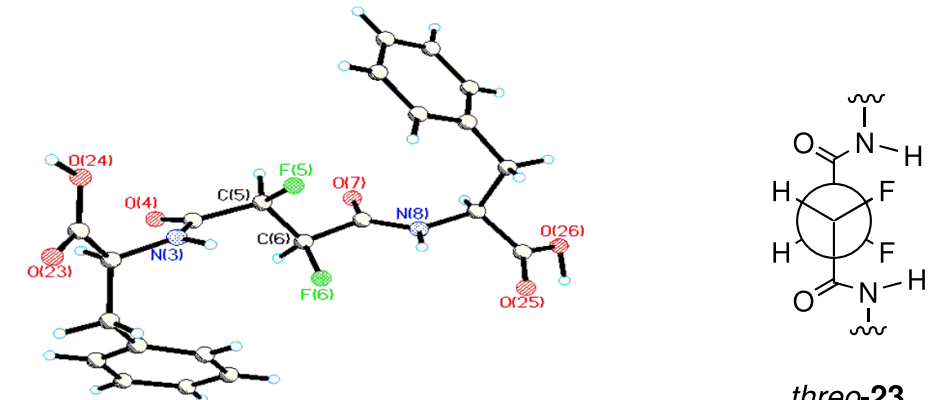

threo-23<smiles>COC(=O)C(Cc1ccccc1)NC(=O)C(F)C(F)C(=O)NC(Cc1ccccc1)C(C)=O</smiles>

erythro-23

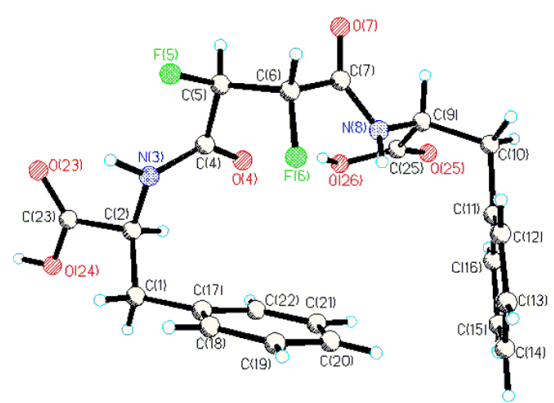

threo-23

Figure 14: The conformations of erythro- and threo- $\mathbf{2 3}$ are very different as a consequence of each conformation preserving a vicinal fluorine gauche relationship [18].

The angular dependence of the ${ }^{3} J_{\mathrm{HF}}$ coupling constant is largely influenced by the electronegativity of the substituents adjacent to the coupling nuclei [31]. For related compounds, the full trans ${ }^{3} J_{\mathrm{HF}}$ coupling constant has been estimated to be approximately $32 \mathrm{~Hz}$ and the gauche ${ }^{3} J_{\mathrm{HF}}$ coupling constant is approximately $8 \mathrm{~Hz}$ [32]. With no conformational bias the average

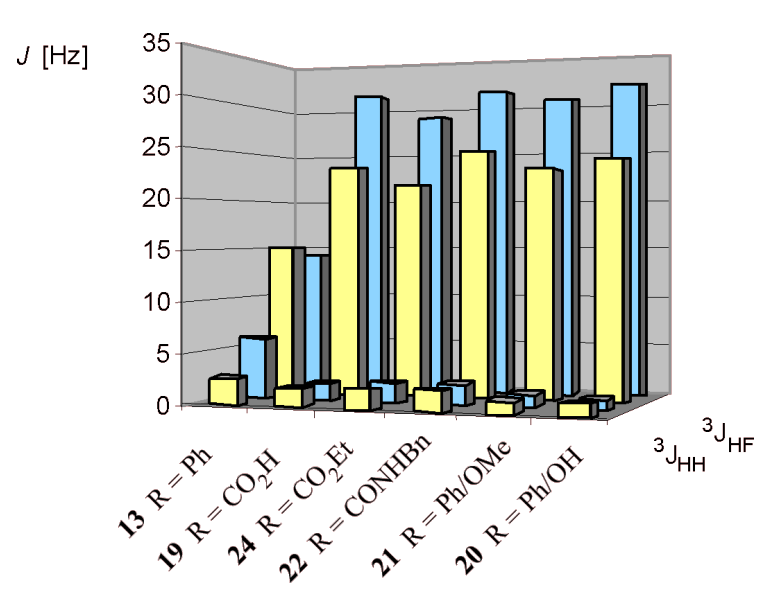

Figure 15: ${ }^{3} \mathrm{~J}_{\mathrm{HF}}$ and ${ }^{3} \mathrm{~J}_{\mathrm{HH}}$ coupling constants for the erythro (yellow) and threo (blue) diastereoisomers of the 2,3-difluorosuccinates 19-22, 24 as well as 1,2-difluoro-1,2-diphenylethanes 13. NMR spectra were recorded in $\mathrm{CDCl}_{3}$ with the exception of 2,3-difluorosuccinic acid, which was measured in $\mathrm{CD}_{3} \mathrm{CN}$. The coupling constants were determined from second order spectra.
${ }^{3} J_{\mathrm{HF}}$ coupling constants will be $(16 \mathrm{~Hz})$ for each of the diastereoisomers according to these values (Figure 17).

The experimental ${ }^{3} J_{\mathrm{HF}}$ coupling constants are clearly different for the two diastereoiomeric series. The contributions of the different conformers can then be estimated from the observed ${ }^{3} J_{\mathrm{HF}}$ NMR coupling constants as illustrated in the equations in Figure 18.

For the erythro diastereoisomer the enantiotopic conformers $\mathbf{b}$ (which will be equally populated), dominate the conformer profile. This is consistent with the observed average ${ }^{3} J_{\mathrm{HH}}$ values of $(2-3 \mathrm{~Hz})$ where in conformers b only $\mathrm{H}-\mathrm{H}$ gauche relationships are found with no contributions from anti $\mathrm{H}-\mathrm{H}$ couplings, which would raise this low value. The high value ${ }^{3} \mathrm{~J}_{\mathrm{HF}}$ of $32 \mathrm{~Hz}$ for the threo diastereoisomers is essentially a maximum value for a trans coupling constant indicating the dominant contribution from conformer $\mathbf{c}$. This is also consistent with the observed average ${ }^{3} J_{\mathrm{HH}}$ values of $(2-3 \mathrm{~Hz})$ where in conformer $\mathbf{c}$ there are only $\mathrm{H}-\mathrm{H}$ gauche relationships.

In overview the dominant conformers in each diastereoisomer series have structures which accommodate gauche relationships between the C-F bonds and these results suggest that the fluorine "gauche effect" is influencing the preferred conformations in solution. It is notable that the coupling constants for the 

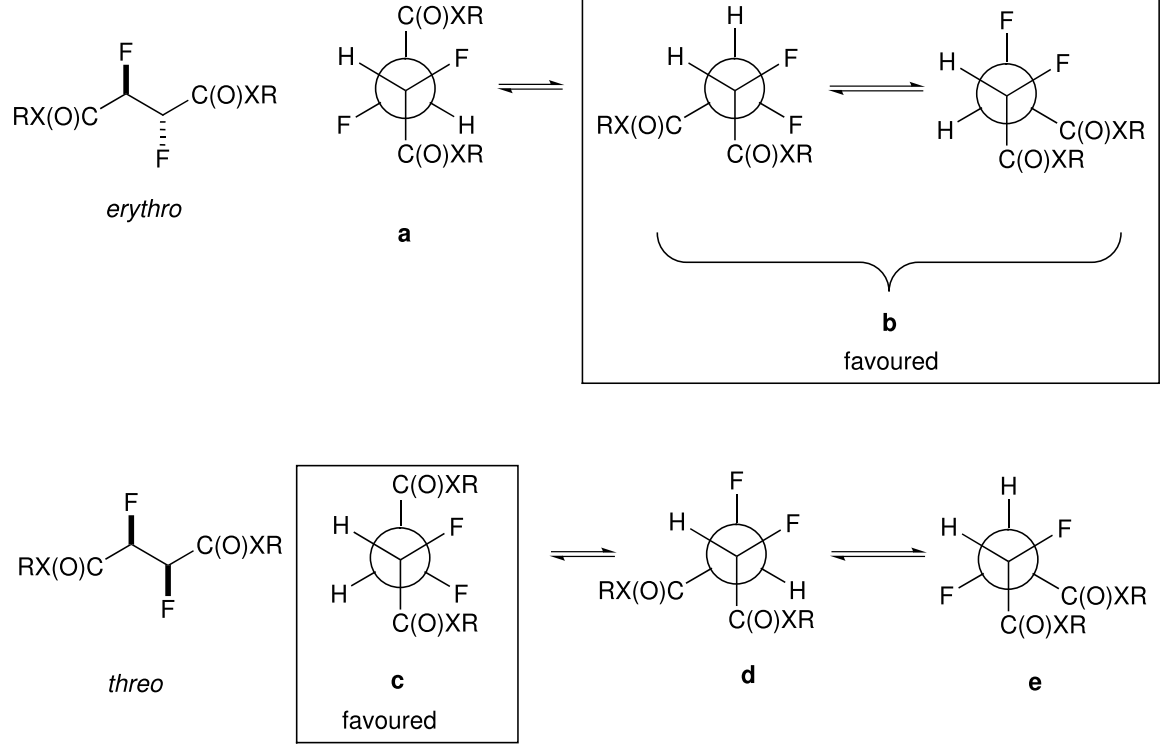

Figure 16: Newman projections of the three staggered conformations of the erythro and threo stereoisomers of the vicinaldifluoro compounds succinates.

$$
\begin{gathered}
\bar{J}_{\mathrm{HF}} \text { (threo) }=\frac{1}{3} \times \frac{\mathrm{J}_{\mathrm{t}}+\mathrm{J}_{\mathrm{t}}}{2}+\frac{1}{3} \times \frac{\mathrm{J}_{\mathrm{g}}+\mathrm{J}_{\mathrm{g}}}{2}+\frac{1}{3} \times \frac{\mathrm{J}_{\mathrm{g}}+\mathrm{J}_{\mathrm{g}}}{2} \sim 16 \mathrm{~Hz} \\
\overline{\mathrm{J}}_{\mathrm{HF}}(\text { erythro })=\frac{1}{3} \times \frac{\mathrm{J}_{\mathrm{g}}+\mathrm{J}_{\mathrm{g}}}{2}+\frac{1}{3} \times \frac{\mathrm{J}_{\mathrm{t}}+\mathrm{J}_{\mathrm{g}}}{2}+\frac{1}{3} \times \frac{\mathrm{J}_{\mathrm{t}}+\mathrm{J}_{\mathrm{g}}}{2} \sim 16 \mathrm{~Hz}
\end{gathered}
$$

Figure 17: The average coupling constant with no conformational bias. The limiting coupling constants $J_{\mathrm{g}}=8 \mathrm{~Hz}$ and $J_{\mathrm{t}}=32 \mathrm{~Hz}$ are estimated values.

$$
\begin{gathered}
\mathrm{J}_{\mathrm{HF}}(\text { erythro })=0 \times \frac{\mathrm{J}_{\mathrm{g}}+\mathrm{J}_{\mathrm{g}}}{2}+0.5 \times \frac{\mathrm{J}_{\mathrm{t}}+\mathrm{J}_{\mathrm{g}}}{2}+0.5 \times \frac{\mathrm{J}_{\mathrm{t}}+\mathrm{J}_{\mathrm{g}}}{2} \sim 20 \mathrm{~Hz} \\
\mathrm{~J}_{\mathrm{HF}}(\text { threo })=1.0 \times \frac{\mathrm{J}_{\mathrm{t}}+\mathrm{J}_{\mathrm{t}}}{2}+0 \times \frac{\mathrm{J}_{\mathrm{g}}+\mathrm{J}_{\mathrm{g}}}{2}+0 \times \frac{\mathrm{J}_{\mathrm{g}}+\mathrm{J}_{\mathrm{g}}}{2} \sim 32 \mathrm{~Hz}
\end{gathered}
$$

Figure 18: The observed ${ }^{3} \mathrm{~J}_{\mathrm{HF}}$ coupling constants are an average over the rotational isomers.

1,2-difluoro-1,2-diphenylethanes isomers $\mathbf{1 3}$ are different in the series and do not conform to the ratios described above.

\section{Conclusion}

In this paper we have described the synthesis and comparative structures of a series of diastereoisomers of vicinal difluoro compounds, which were generated by converting stilbenes to 1,2-difluoro,1-2-diphenylethanes $\mathbf{1 3}$ and then oxidation of the aryl rings to generate 2,3-difluorosuccinic acids and their derivatives. The preparative methods allowed the preparation of individual erythro or threo diastereoisomers. The tendency of the vicinal fluorines to adopt predominant gauche conformations in solution emerges from an analysis of vicinal ${ }^{3} J_{\mathrm{HH}}$ and ${ }^{3} J_{\mathrm{HF}}$ coupling constants of these molecules and reinforces earlier studies on the conformation of vicinal difluoro compounds. This is in line with the well described fluorine gauche effect. The only exception to this was found for the threo stereoisomer of 1,2-difluoro-1,2-diphenylethanes $\mathbf{1 3}$, where all of the data (ab initio, NMR and X-ray) did not converge on a consensus structure. It emerges from this study that the stereoselective incorporation of vicinal fluorines can be used to influence the conformation of organic molecules. This is an attractive tool for the design of performance molecules in areas as diverse as pharmaceutical and medicinal chemistry research to materials science.

Experimental details for the preparation and characterisation of compounds 13, 14, 15, 19, 21, 22 and 24 are given in Supporting Information File 1.

\section{Supporting Information}

\section{Supporting Information File 1}

Experimental and characterisation details of synthesised compounds.

[http://www.beilstein-journals.org/bjoc/content/ supplementary/1860-5397-2-19-S1.rtf]

\section{Supporting Information File 2}

Calculated confirmations and energies.

[http://www.beilstein-journals.org/bjoc/content/ supplementary/1860-5397-2-19-S2.zip] 


\section{Acknowledgments}

We thank the University of St Andrews for Studentship support (MS) and AMZS thanks the EPSRC for financial support. We also thank Professor Raymond Abrahams of the University of Liverpool for useful discussions on NMR interpretation.

\section{References}

1. Craig, N. C.; Chen, A.; Suh, K. H.; Klee, S.; Mellau, G. C.; Winnewisser, B. P.; Winnewisser, M. J. Am. Chem. Soc. 1997, 119, 4789-4790. doi:10.1021/ja963819e

2. Angelini, G.; Gavuzzo, E.; Segre, A. L.; Speranza, M. J. Phys. Chem. 1990, 94, 8762-8766. doi:10.1021/j100388a004

3. Tavasli, M.; O'Hagan, D.; Pearson, C.; Petty, M. C. Chem. Commun. 2002, 1226-1227. doi:10.1039/b202891c

4. Merritt, R. F. J. Am. Chem. Soc. 1967, 89, 609-612. doi:10.1021/ ja00979a025

5. Chia, T.; Yang, N. C.; Chernick, C. L. J. Am. Chem. Soc. 1964, 86, 5021-5022. doi:10.1021/ja01076a069

6. Burmakov, A. I.; Motnyak, L. A.; Kunshenko, B. V.; Alexeeva, L. A.; Yagupolskii, L. M. J. Fluorine Chem. 1981, 19, 151-161. doi:10.1016/ S0022-1139(00)81331-7

7. Hudlicky, M. J. Fluorine Chem. 1983, 23, 241-259. doi:10.1016/S00221139(00)85130-1

8. Singh, R. P.; Shreeve, J. M. J. Fluorine Chem. 2002, 116, 23-26. doi:10.1016/S0022-1139(02)00065-9

9. Lal, G. S.; Pez, G. P.; Pesaresi, R. J.; Prozonic, F. M. Chem. Commun. 1999, 215-216. doi:10.1039/a808517j

10. Lal, G. S.; Pez, G. P.; Pesaresi, R. J.; Prozonic, F. M.; Cheng, H. J. Org. Chem. 1999, 64, 7048-7054. doi:10.1021/jo990566+

11. Hulin, B.; Cabral, S.; Lopaze, M. G.; Van Volkenburg, M. A.; Andrews, K. M.; Parker, J. C. Bioorg. Med. Chem. Lett. 2005, 15, 4770-4773. doi:10.1016/j.bmcl.2005.07.026

12. Caldwell, C. G.; Chen, P.; He, J.; Parmee, R. E.; Leiting, B.; Marsilio, F.; Patel, R. A.; Wu, J. K.; Eiermann, G. J.; Petrov, A.; He, H.; Lyons, K. A.; Thornberry, N. A.; Weber, A. E. Bioorg. Med. Chem. Lett. 2004, 14, 1265-1268. doi:10.1016/j.bmcl.2003.12.040

13. Hamatani, T.; Matsubara, S.; Matsuda, H.; Schlosser, M. Tetrahedron 1988, 44, 2875-2881. doi:10.1016/S0040-4020(88)90023-3

14. Lal, G. S.; Labach, E.; Evans, A. J. Org. Chem. 2000, 65, 4830-4832. doi:10.1021/jo000020j

15. Olah, G. J. Org. Chem. 1979, 44, 3872-3881. doi:10.1021/ jo01336a027

16. Rozen, S. J. Org. Chem. 1986, 51, 3607-3611. doi:10.1021/ jo00369a011

17. Norsikian, S. Chem.-Eur. J. 1999, 5, 2055-2068. doi:10.1002/(SICI) 1521-3765(19990702)5:7<2055::AID-CHEM2055>3.0.CO;2-9

18. Schüler, M.; O'Hagan, D.; Slawin, A. M. Z. Chem. Commun. 2005, 4324-4326. doi:10.1039/b506010a

19. Olah, G.; Nojima Keres, M. I. Synthesis 1973, 780-783. doi:10.1055/s1973-22298

20. Ernet, E.; Haufe, G. Synthesis 1997, 953-956. doi:10.1055/s-19971279

21. Barton, D. H. R. J. Chem. Soc., Perkin Trans. 1 1974, 739-742. doi:10.1039/p19740000739

22. Harwood, L. Polar Rearrangements; Oxford Chemistry Primers; Oxford University Press: Tokyo, 1992.

23. Abraham, R. J.; Loftus, P. Tetrahedron 1977, 33, 1227-1234. doi:10.1016/0040-4020(77)80419-5

24. Gaussian03, Revision C.02; Gaussian, Inc.: Wallingford, CT, 2004.
25. Kendall, R. A.; Dunning, T. H., Jr.; Harrison, R. J. J. Chem. Phys. 1992, 96, 6796-6806. doi:10.1063/1.462569

26. Barone, V.; Cossi, M. J. Phys. Chem. A 1998, 102, 1995-2001. doi:10.1021/jp9716997

27. Aoyama, T. J. Chem. Soc., Perkin Trans. 1 1995, 15, 1905-1912. doi:10.1039/p19950001905

28. Nakajima, M.; Tomioka, K.; Koga, K. Tetrahedron 1993, 49, 9735-9750. doi:10.1016/S0040-4020(01)80176-9

29. Banks, J. W.; Batsanov, A. S.; Howard, J. A. K.; O'Hagan, D.; Rzepa, H. S.; Martin-Santamaria, S. J. Chem. Soc., Perkin Trans. 2 1999, 2409-2411. doi:10.1039/a907452j

30. Reed, A. E.; Curtiss, L. A.; Weinhold, F. Chem. Rev. 1988, 88, 899-926. doi:10.1021/cr00088a005

31. Abraham, R. J.; Cavalli, L. Mol. Phys. 1965, 9, 67. doi:10.1080/ 00268976500100091

32. Ihrig, A. M.; Smith, S. L. J. Am. Chem. Soc. 1972, 94, 34-41. doi:10.1021/ja00756a007

\section{License and Terms}

This is an Open Access article under the terms of the Creative Commons Attribution License

(http://creativecommons.org/licenses/by/2.0), which permits unrestricted use, distribution, and reproduction in any medium, provided the original work is properly cited.

The license is subject to the Beilstein Journal of Organic Chemistry terms and conditions:

(http://www.beilstein-journals.org/bjoc)

The definitive version of this article is the electronic one which can be found at: doi:10.1186/1860-5397-2-19 